$\underline{\text { articles }}$

\title{
Rural Entrepreneurship Success Factors: An Empirical Investigation in an Emerging Market
}

\author{
Prince Gyimah ${ }^{1}$, ${ }^{\text {Robert N. Lussier }}{ }^{2}$ \\ ${ }^{1}$ Department of Accounting Studies Education, Akenten Appiah-Menka University of Skills Training and Entrepreneurial Development, ${ }^{2}$ Department \\ of Business Management, Springfield College \\ Keywords: Rural Entrepreneurs, Success, Failure, Sustainable Development, Emerging Market, Ghana \\ https://doi.org/10.53703/001c.29470
}

\section{Journal of Small Business Strategy}

Vol. 31, Issue 4, 2021

\begin{abstract}
Small businesses in rural communities play a key role in achieving global sustainable economic development because they are the driving force of poverty reduction, job creation, resiliency, and economic development. This study examines the factors that drive the success or failure of small businesses in rural communities in an emerging market. The methodology is survey interview research using a logistic regression model to test the Lussier success vs failure prediction model with a sample of 230 businesses (successful $n=120$, failed $n=110$ ) from the rural communities in an emerging market. This study supports the Lussier model validity $(\mathrm{p}<0.01)$ with a high overall accuracy of $71 \%$ in predicting a venture as successful or failed. Capital, industry experience, staffing, and marketing skills are the most significant (t-values $<.05)$ factors that distinguish successful from failed rural businesses in an emerging market. The findings can help future, and nascent rural entrepreneurs avoid failure and successfully contribute to economic development. Implications for government agencies, public regulatory bodies, financial institutions, investors, suppliers, educators, professional institutions, and society, as well as limitations and future research, are presented. This study also contributes to the international validity of the Lussier model that can be used in both advanced and developing economies, and it contributes to the development of theory.
\end{abstract}

\section{Introduction}

Extreme poverty in rural economies is one of the world's most pressing problems, and more governments and other organizations are turning to entrepreneurship to grow rural economies (Abebe \& Gebremariam, 2021; Yeboah-Asiamah et al., 2015). Why entrepreneurship and why rural entrepreneurship? Rural entrepreneurs are different from other types of entrepreneurs, as they utilize and develop local resources to grow the local economy (Korsgaard et al., 2015; Müller \& Korsgaard, 2018; Soleymani et al., 2021). Small businesses in rural communities play a key role in achieving global sustainable economic development because they are the driving force of poverty reduction, job creation, resiliency, and economic development (Achua \& Lussier, 2014; Blankson et al., 2018; Boohene \& Agyapong, 2017; A. Crawford \& Barber, 2020; Fortunato, 2014; Paynter et al., 2021). For instance, rural entrepreneurs in emerging markets employ $60 \%$ of the workforce (Borchardt et al., 2018) and contribute over $50 \%$ to the Gross Domestic Product
(Oppong et al., 2014).

Although rural small businesses play a significant role in emerging markets, most do not survive for more than five years and have a high failure rate (Deller \& Conroy, 2017; Nikolić et al., 2019). Therefore, the prediction of business failure or success has become an imperative research topic to entrepreneurship, and other relevant stakeholders such as international and government agencies, public policymakers, investors, suppliers, educators, and consultants (Dennis \& Fernald, 2001; Galli-Debicella, 2020; Gyimah et al., 2019, 2020; Gyimah \& Adeola, 2021; Halabí \& Lussier, 2014; Lussier \& Corman, 1995). Thus, knowing the critical factors that contribute to the success or failure of rural businesses is crucial to the attainment of sustainable development (Salder et al., 2020).

However, the literature reveals a great inconsistency of the factors contributing to the success or failure of businesses (Abebe \& Gebremariam, 2021). To date, there is no theory (Adeola et al., 2021; Lussier et al., 2016; Marom \& Lussier, 2014). There is a need for further research to under-

\footnotetext{
a Akenten Appiah-Menka University of Skills Training and Entrepreneurial Development, P. O. Box 1277, Kumasi, Ghana, princegyima@yahoo.co.uk

b Springfield College, Springfield, MA 01109, USA rlussier@springfieldcollege.edu Corresponding Author
} 
stand better and predict the success versus failure of rural businesses due to their significant role in emerging markets (Baidoun et al., 2019; Hyder \& Lussier, 2016). The purpose of this study is to examine the factors that drive the success or failure of small businesses in rural communities in the emerging market of Ghana that has over $43 \%$ of the total population living in rural areas.

The study contributes to the theoretical literature and provides practical implications for entrepreneurs and public policymakers. For instance, the study contributes to the understanding of the critical success factors of businesses that can reduce the failure rate in emerging markets. It, therefore, has practical implications for start-up ventures and established small business owners and managers. Also, government agencies, public policymakers, investors, suppliers, educators, and consultants can use the model to aid in their decisions. Moreover, this study has practical implications that can also strengthen the businesses by contributing to a holistic approach in achieving the United Nations Sustainable Development Goals (SDG); including no poverty (SDG 1); and decent work, and economic growth (SDG 8). Finally, since there is no universal theory or model for predicting small business success or failure (Lussier \& Pfeifer, 2000; Marom \& Lussier, 2014), this study further validates the Lussier (1995) model as an international predictor of small business success or failure that can be used in other developing and developed countries.

\section{Literature Review Rural Entrepreneurship Context}

Poverty, unemployment, and lack of economic growth remain daunting issues for most developing economies, especially in Sub-Saharan Africa (Abebe \& Gebremariam, 2021). To solve these challenges, support for rural entrepreneurship is needed (Soleymani et al., 2021; Yeboah-Asiamah et al., 2015). Eighty-seven years after Joseph Schumpeter's seminal book on the economic development theory, entrepreneurship continues to dominate business literature because of its immense contribution to poverty eradication, employment, economic development, and growth (Abebe \& Gebremariam, 2021; Barber et al., 2019; Blankson et al., 2018; A. Crawford \& Barber, 2020; Fortunato, 2014; Pato \& Teixeira, 2018).

Despite the significant importance of entrepreneurship, there is no universally accepted definition of entrepreneurship due to its multifaceted context. Thus, rural entrepreneurship has diverse definitions and meanings (Pato \& Teixeira, 2018; Saadatmand \& Barber, 2019). Wortman (1990) first defined rural entrepreneurship as "the creation of a new organization that introduces a new product, serves or creates a new market, or utilizes a new technology in a rural environment.” McElwee \& Smith (2014) further defined it as firms that engage indigenous individuals, deploy indigenous services and generate revenue for the rural environment.

The rural entrepreneurship concept advocates that the activities of businesses should add value to the socio-spatial aspects of the rural regions (Pato \& Teixeira, 2018). This is attained by developing new value from local resources and linking to the features and rareness of the indigenous to produce goods with value (Pato \& Teixeira, 2018). The study focuses on the creation and development of micro, small and medium businesses in a rural setting that is community-based that uses local resources in their business activities. Despite an emergent prominence on rural entrepreneurship, how rural enterprises grow remains uncertain (Deller et al., 2019), and very little is known about rural entrepreneurship (Muñoz \& Kimmitt, 2019; Pato \& Teixeira, 2018).

Extant literature on rural entrepreneurship in advanced economies focused on agricultural perspective, skills requirement for local economic business, gender and family studies, farm diversification, and social capital (Koyana \& Mason, 2017). However, studies on rural entrepreneurship investigated policy application on how rural areas can be developed in emerging markets, focusing on women and youth (Koyana \& Mason, 2017). This present study extends the literature by examining the critical factors that contribute to the success or failure of rural enterprises in an emerging market, an important but neglected area of research.

\section{Success vs Failure Variables}

A number of studies have investigated the contributing factors to the success or failure of businesses in both developing and developed countries (Ciampi et al., 2021; Habersetzer et al., 2021). In developed countries, Habersetzer et al. (2021) concluded that industry experiences contribute to the success of firms in rural regions. Skuras et al. (2005) also found that human capital accumulation practices such as managerial experiences or education and training predict business success in rural communities. Halabí \& Lussier (2014) found that adequate capital, record keeping, and financial controls, use of the internet and professional advice, owners having parents owning a business, partners, and marketing skills contribute to the success or failure of businesses. Blackwood \& Mowl (2000) also found that the success or failure of businesses depends on the behavior of business owners, social behaviors, and the economic factors of the environment.

Other scholarly researchers in developed economies assert that entrepreneurial characteristics such as owner's age (Barkham et al., 1996; Bosma et al., 2000; Bruins et al., 2000; Cooper et al., 1990; Duchesneau \& Gartner, 1990; Kangasharju, 2000; Lussier, 1995; Lussier \& Pfeifer, 2001; Marom \& Lussier, 2014; Storey, 1994); culture, external business factors, technology (Peake et al., 2019); managerial skills, experience, training and business environment (Barsley \& Kleiner, 1990; Covin \& Covin, 1990; Dess et al., 1997; Dun \& Bradstreet, 1995; Postma \& Zwart, 2001), owner's competencies and quality (Miskin \& Rose, 1990), and innovation (Dunne et al., 2016) contribute to the success or failure of businesses.

For developing economies, environmental conditions and entrepreneurial skills are the key determinants of the performance of small businesses (Benzing et al., 2005; Chu et al., 2007; Ghosh et al., 1993; Huck \& McEwen, 1991; Yusuf, 1995). Financial support, demographic factors, age at which the new business venture is undertaken, use of family loans, and the initial size of the firm are all instrumental 
1. Capital (capt). Businesses that start undercapitalized have a greater chance of failure than firms that start with adequate capital.

2. Record keeping and financial control ( $r$ kfc). Businesses that do not keep updated and accurate records and do not use adequate financial controls have a greater chance of failure than firms that do.

3. Industry Experience (inex). Businesses managed by people without prior industry experience have a greater chance of failure than firms managed by people with prior industry experience.

4. Management Experience (maex). Businesses managed by people without prior management experience have a greater chance of failure than firms managed by people with prior management experience.

5. Planning (plan). Businesses that do not develop specific business plans have a greater chance of failure than firms that do.

6. Professional Advisors (prad). Businesses that do not use professional advisors have a greater chance of failure than firms using professional advisors. More recent sources of professional advisors are venture capitalists.

7. Education (educ). People without any college education who start a business have a greater chance of failing than people with one or more years of college education.

8. Staffing (staff). Businesses that cannot attract and retain quality employees have a greater chance of failure than firms that can.

9. Product/Service Timing (psti). Businesses that select products/services that are too new or too old have a greater chance of failure than firms that select products/services that are in the growth stage.

10. Economic Timing (ecti). Businesses that start during a recession have a greater chance of failing than firms that start during expansion periods.

11. Age (age). Younger people who start a business have a greater chance of failing than older people starting a business.

12. Partners (part). A business started by one person has a greater chance of failure than a firm started by more than one person.

13. Parents (pent). Business owners whose parents did not own a business have a greater chance of failure than owners whose parents did own a business.

14. Minority (mior). Minorities have a greater chance of failure than non-minorities.

15. Marketing (mrkt). Business owners without marketing skills have a greater chance of failure than owners with marketing skills.

in subsequent business success. Adeola et al. (2021) found that for businesses in developing countries to be successful, entrepreneurs should start with adequate capital, have marketing skills, keep financial records, and follow professional advice.

Similarly, Baidoun et al. (2019) reported that capital, record keeping, and financial controls, and the use of professional advice are the drivers of the success or failure of businesses in emerging markets. Neshamba (2000) also found that previous managerial experience, understanding of customers' needs, capital accessibility, and hard work contribute to the success of a business. The literature reveals a great inconsistency of the contributing factors to the firm's success or failure, and to date, there is no theory (Adeola et al., 2021; Lussier et al., 2016). With the lack of consistency of success versus failure variables, there is a need for further research to understand better and predict the success versus failure of rural businesses in emerging markets.

\section{Prediction Models}

The last three decades have provided quantitative mod- els for predicting the success or failure of businesses. Failure prediction models are based on large public firms due to the availability and accessibility of financial data (Gyimah et al., 2020). Financial data, however, can be manipulated using some accounting policies, and it is unrealistic to use financial information to predict the failure or success of a business (Rosner, 2003). Financial models also use prior sales and profits to predict failure. However, start-up firms have no financial history, and thus, financial models cannot be used with nascent firms. Therefore, researchers are calling for non-financial prediction models that can better predict the success or failure of businesses (Appiah, 2011; Appiah et al., 2015; Gyimah \& Boachie, 2018).

This study adopts the Lussier (1995) model that has been validated for the prediction of success or failure of businesses (Halabí \& Lussier, 2014; Teng et al., 2011). The Lussier (1995) model consists of 15 non-financial variables that were selected from 20 prior studies identified as contributing factors to the success and failure of small businesses. Table 1 includes the Lussier (1995) success versus failure prediction variables. There are now 44 articles discussing the variables in the model; see Table 2 . 
Table 2. A comparison of variables identified in $\mathbf{4 4}$ articles as contributing factors to business success versus failure

\begin{tabular}{|c|c|c|c|c|c|c|c|c|c|c|c|c|c|c|c|}
\hline Scholars & Capt & rkfc & Inex & Maex & Plan & prad & Educ & Staff & Psti & ecti & age & part & pent & minor & mrkt \\
\hline Adeola et al., 2021 & $\mathrm{~F}$ & $\mathrm{~F}$ & $\mathrm{~N}$ & N & $\mathrm{N}$ & $\mathrm{F}$ & $\mathrm{N}$ & N & $\mathrm{F}$ & N & $\mathrm{N}$ & $\mathrm{N}$ & $\mathrm{F}$ & $\mathrm{N}$ & $\mathrm{N}$ \\
\hline Baidoun et al., 2019 & $\mathrm{~F}$ & $\mathrm{~F}$ & $\mathrm{~N}$ & N & $\mathrm{F}$ & $\mathrm{F}$ & $\mathrm{N}$ & N & $\mathrm{N}$ & N & N & $\mathrm{N}$ & $\mathrm{N}$ & $\mathrm{N}$ & $\mathrm{N}$ \\
\hline Barsley \& Welner, 1990 & $\mathrm{~F}$ & - & $\mathrm{F}$ & $\mathrm{F}$ & $\mathrm{F}$ & $\mathrm{F}$ & - & - & - & - & - & - & - & - & - \\
\hline Bosma et al., 2000 & - & - & $\mathrm{F}$ & - & - & - & - & - & - & - & $\mathrm{F}$ & - & - & - & - \\
\hline Bruins et al., 2000 & $\mathrm{~F}$ & - & $\mathrm{F}$ & N & - & - & $\mathrm{N}$ & - & - & - & $\mathrm{F}$ & - & - & - & - \\
\hline Bruno et al., 1987 & $\mathrm{~F}$ & $\mathrm{~F}$ & - & $\mathrm{F}$ & $\mathrm{F}$ & - & - & $\mathrm{F}$ & $\mathrm{F}$ & $\mathrm{F}$ & - & - & - & - & $\mathrm{F}$ \\
\hline Carrero-Morales, 2015 & N & $N$ & $N$ & N & $\mathrm{N}$ & $N$ & $\mathrm{~N}$ & $\mathrm{~F}$ & $\mathrm{~F}$ & $\mathrm{~N}$ & N & $\mathrm{N}$ & $\mathrm{N}$ & $\mathrm{F}$ & $\mathrm{N}$ \\
\hline Cooper et al., 1990 & $\mathrm{~F}$ & - & $\mathrm{N}$ & $N$ & $\mathrm{~F}$ & $\mathrm{~F}$ & $\mathrm{~N}$ & - & $\mathrm{F}$ & $\mathrm{F}$ & $\mathrm{F}$ & $\mathrm{F}$ & - & $\mathrm{F}$ & - \\
\hline Cooper et al., 1991 & $\mathrm{~F}$ & - & $\mathrm{F}$ & N & - & $\mathrm{F}$ & $\mathrm{F}$ & - & $\mathrm{N}$ & $N$ & N & $\mathrm{N}$ & $\mathrm{F}$ & $\mathrm{F}$ & - \\
\hline Crawford, 1974 & - & - & $\mathrm{F}$ & - & - & $\mathrm{F}$ & $\mathrm{F}$ & - & - & N & $\mathrm{N}$ & - & - & - & - \\
\hline Cressy, 1996 & $\mathrm{~F}$ & - & - & $\mathrm{F}$ & - & - & - & - & - & - & - & - & - & - & - \\
\hline Dun \& Bradstreet, 1995 & $\mathrm{~F}$ & $\mathrm{~F}$ & $\mathrm{~F}$ & $\mathrm{~F}$ & - & - & - & - & - & $\mathrm{F}$ & - & - & - & - & - \\
\hline Flahvin, 1985 & $\mathrm{~F}$ & $\mathrm{~F}$ & $\mathrm{~F}$ & $\mathrm{~F}$ & - & $\mathrm{F}$ & - & $\mathrm{F}$ & - & - & - & - & - & - & - \\
\hline Gaskill et al., 1993 & N & $\mathrm{F}$ & $\mathrm{F}$ & $\mathrm{F}$ & $\mathrm{F}$ & $\mathrm{F}$ & $\mathrm{N}$ & - & - & $\mathrm{N}$ & - & - & - & - & $\mathrm{F}$ \\
\hline Guzman \& Lussier, 2015 & N & $N$ & $\mathrm{~N}$ & N & $\mathrm{N}$ & $N$ & $\mathrm{~N}$ & N & $\mathrm{N}$ & $N$ & $N$ & $\mathrm{~N}$ & $\mathrm{~N}$ & $\mathrm{~N}$ & $\mathrm{~N}$ \\
\hline Gyimah \& Adeola, 2021 & $\mathrm{~F}$ & $N$ & $\mathrm{~N}$ & N & $\mathrm{F}$ & $N$ & $\mathrm{~N}$ & $N$ & $\mathrm{~N}$ & $\mathrm{~N}$ & $N$ & $\mathrm{~N}$ & $\mathrm{~N}$ & $\mathrm{~N}$ & $\mathrm{~F}$ \\
\hline Gyimah et al., 2020 & $\mathrm{~F}$ & $N$ & $\mathrm{~N}$ & N & $\mathrm{N}$ & $N$ & $\mathrm{~N}$ & N & $\mathrm{N}$ & $\mathrm{F}$ & $N$ & $\mathrm{~N}$ & $\mathrm{~N}$ & $\mathrm{~N}$ & $\mathrm{~F}$ \\
\hline Hoad \& Rosko, 1964 & - & - & $\mathrm{F}$ & N & $\mathrm{N}$ & $\mathrm{F}$ & $\mathrm{F}$ & - & - & - & - & - & - & - & - \\
\hline Houben et al., 2005 & N & $N$ & $\mathrm{~N}$ & $\mathrm{~F}$ & $\mathrm{~F}$ & $\mathrm{~F}$ & $\mathrm{~N}$ & N & $\mathrm{F}$ & $\mathrm{F}$ & $N$ & $\mathrm{~F}$ & $\mathrm{~N}$ & $\mathrm{~N}$ & $\mathrm{~F}$ \\
\hline Hyder \& Lussier, 2016 & $\mathrm{~F}$ & $N$ & $\mathrm{~N}$ & N & $\mathrm{F}$ & N & $\mathrm{N}$ & $\mathrm{F}$ & $\mathrm{N}$ & $N$ & $N$ & $\mathrm{~F}$ & $\mathrm{~N}$ & N & $N$ \\
\hline Kennedy, 1985 & $\mathrm{~F}$ & - & - & $\mathrm{F}$ & $\mathrm{F}$ & - & - & - & - & $\mathrm{F}$ & - & - & - & - & - \\
\hline Lauzen, 1985 & $\mathrm{~F}$ & $\mathrm{~F}$ & - & $\mathrm{F}$ & $\mathrm{F}$ & - & - & $\mathrm{F}$ & - & - & - & - & - & - & - \\
\hline Lussier \& Corman, 1996 & $\mathrm{~F}$ & $\mathrm{~F}$ & $\mathrm{~F}$ & N & $\mathrm{F}$ & $\mathrm{F}$ & $\mathrm{F}$ & $\mathrm{F}$ & $\mathrm{N}$ & $\mathrm{F}$ & $N$ & $\mathrm{~N}$ & $\mathrm{~F}$ & $\mathrm{~F}$ & $\mathrm{~N}$ \\
\hline Lussier \& Halabi, 2010 & N & $N$ & $\mathrm{~N}$ & N & $\mathrm{N}$ & $N$ & $\mathrm{~N}$ & N & $\mathrm{N}$ & $N$ & N & $\mathrm{N}$ & $\mathrm{N}$ & N & $N$ \\
\hline Lussier \& Pfeifer, 2001 & N & $N$ & $\mathrm{~N}$ & N & $\mathrm{F}$ & $\mathrm{F}$ & $\mathrm{F}$ & $\mathrm{F}$ & $\mathrm{N}$ & $N$ & N & $\mathrm{N}$ & $\mathrm{N}$ & N & $\mathrm{N}$ \\
\hline Lussier, 1995 & N & $N$ & $N$ & N & $\mathrm{F}$ & $\mathrm{F}$ & $\mathrm{F}$ & N & $N$ & $N$ & $N$ & $\mathrm{~F}$ & $\mathrm{~N}$ & N & $N$ \\
\hline Lussier, 1996a & $N$ & $\mathrm{~F}$ & $N$ & $\mathrm{~F}$ & $\mathrm{~F}$ & $\mathrm{~F}$ & $\mathrm{~N}$ & $\mathrm{~F}$ & $N$ & $\mathrm{~F}$ & $N$ & $\mathrm{~F}$ & $\mathrm{~F}$ & $\mathrm{~N}$ & $\mathrm{~F}$ \\
\hline Lussier, 1996b & $\mathrm{N}$ & $\mathrm{F}$ & $N$ & N & $\mathrm{F}$ & $\mathrm{F}$ & $\mathrm{N}$ & N & $\mathrm{F}$ & $\mathrm{F}$ & $\mathrm{F}$ & $\mathrm{N}$ & $\mathrm{N}$ & $\mathrm{N}$ & $N$ \\
\hline Lussier et al., 2016 & N & $\mathrm{F}$ & $\mathrm{N}$ & N & $\mathrm{F}$ & - & $\mathrm{N}$ & $\mathrm{F}$ & $\mathrm{F}$ & - & - & $\mathrm{N}$ & - & - & $\mathrm{F}$ \\
\hline
\end{tabular}




\begin{tabular}{|c|c|c|c|c|c|c|c|c|c|c|c|c|c|c|c|}
\hline Scholars & Capt & rkfc & Inex & Maex & Plan & prad & Educ & Staff & Psti & ecti & age & part & pent & minor & mrkt \\
\hline Marom \& Lussier, 2014 & $\mathrm{~F}$ & $\mathrm{~F}$ & $N$ & $\mathrm{~N}$ & $\mathrm{~F}$ & $\mathrm{~F}$ & $\mathrm{~N}$ & $\mathrm{~N}$ & $N$ & $\mathrm{~N}$ & $\mathrm{~F}$ & $N$ & $\mathrm{~N}$ & $N$ & $N$ \\
\hline Mcqueen, 1989 & $\mathrm{~F}$ & - & $\mathrm{F}$ & $\mathrm{F}$ & - & - & - & - & - & - & - & - & - & - & $\mathrm{F}$ \\
\hline Rauch et al., 2005 & - & - & $\mathrm{F}$ & $\mathrm{F}$ & - & - & $\mathrm{F}$ & - & - & - & - & - & - & - & - \\
\hline Reynolds \& Miller, 1989 & $\mathrm{~F}$ & $\mathrm{~F}$ & - & - & $\mathrm{F}$ & - & $\mathrm{N}$ & $\mathrm{N}$ & $\mathrm{F}$ & - & $\mathrm{N}$ & $\mathrm{F}$ & - & - & - \\
\hline Reynolds, 1987 & $\mathrm{~F}$ & $\mathrm{~F}$ & - & - & $\mathrm{F}$ & - & - & $\mathrm{N}$ & $\mathrm{F}$ & - & - & - & - & - & $\mathrm{N}$ \\
\hline Sage, 1993 & $\mathrm{~F}$ & - & - & $\mathrm{F}$ & - & - & $\mathrm{F}$ & - & - & - & - & - & - & - & - \\
\hline Santarelli, 1998 & - & - & - & - & - & - & $\mathrm{F}$ & - & - & $\mathrm{F}$ & - & - & - & - & - \\
\hline Schutjens \& Weaver, 2000 & - & - & - & - & $\mathrm{F}$ & - & - & - & - & - & - & $\mathrm{F}$ & - & - & - \\
\hline Sommers \& Koc, 1987 & - & - & - & $\mathrm{F}$ & $\mathrm{F}$ & - & - & $\mathrm{F}$ & - & - & - & - & - & - & - \\
\hline Teng et al., 2011 & $\mathrm{~N}$ & $\mathrm{~N}$ & $\mathrm{~N}$ & $\mathrm{~N}$ & $\mathrm{~N}$ & $\mathrm{~N}$ & $\mathrm{~N}$ & $\mathrm{~N}$ & $\mathrm{~F}$ & $\mathrm{~N}$ & $\mathrm{~N}$ & N & $\mathrm{N}$ & $\mathrm{N}$ & $\mathrm{F}$ \\
\hline Thompson, 1988 & $\mathrm{~N}$ & - & - & $\mathrm{F}$ & $\mathrm{F}$ & - & - & $\mathrm{F}$ & $\mathrm{F}$ & - & - & - & - & - & - \\
\hline Vesper, 1990 & $\mathrm{~F}$ & $\mathrm{~F}$ & $\mathrm{~F}$ & $\mathrm{~F}$ & $\mathrm{~N}$ & $\mathrm{~F}$ & $\mathrm{~F}$ & - & $\mathrm{F}$ & $\mathrm{F}$ & - & $\mathrm{F}$ & - & - & $\mathrm{F}$ \\
\hline Wight, 1985 & $\mathrm{~F}$ & $\mathrm{~F}$ & - & $\mathrm{F}$ & - & $\mathrm{F}$ & - & - & - & - & - & - & - & - & - \\
\hline Wiklund \& Shepherd, 2003 & $\mathrm{~F}$ & - & - & $\mathrm{F}$ & - & - & - & - & - & - & - & - & - & - & - \\
\hline Wood, 1989 & - & $\mathrm{F}$ & $\mathrm{F}$ & $\mathrm{F}$ & $\mathrm{F}$ & - & $\mathrm{F}$ & - & - & - & $\mathrm{F}$ & - & - & - & - \\
\hline Total F & 24 & 16 & 14 & 19 & 23 & 18 & 11 & 11 & 12 & 11 & 5 & 8 & 4 & 4 & 10 \\
\hline Total N & 12 & 11 & 17 & 19 & 8 & 7 & 18 & 13 & 12 & 14 & 17 & 14 & 13 & 14 & 12 \\
\hline Total - & 8 & 17 & 13 & 6 & 13 & 19 & 15 & 20 & 20 & 19 & 22 & 22 & 27 & 26 & 22 \\
\hline
\end{tabular}

$F$ supports variable as a contributing factor.

$N$ does not support variable as a contributing factor.

does not mention varible as a contibuting foctor. 


\section{Methods}

\section{Sample and Model}

This survey interview research study uses quantitative analysis to test the Lussier success vs failure prediction model. The study adopts the Lussier (1995) model questionnaire that has been previously validated in multiple studies. Following Adeola et al. (2021); Lussier and Halabi’s (2010) studies, this study uses a simple random sampling technique to select rural businesses in Ghana. Trained professionals administered 500 questionnaires to local businesses in the rural communities; however, 230 completed the survey resulting in a $46 \%$ response rate. Of the respondents, 120 are categorized as successful and 110 as failed businesses.

This study replicates previous studies using binary logistic regression to test the Lussier (1995) prediction model variables (e.g., Adeola et al., 2021; Gyimah et al., 2020; Hyder \& Lussier, 2016; Lussier, 1995; Lussier et al., 2016; Lussier \& Halabi, 2010; Teng et al., 2011). The model is:

Log $($ success $/$ failure $)=\delta_{0}+\delta_{1}$ Capital

$+\delta_{2}$ Record keeping financial control

$+\delta_{3}$ Industry experience

$+\delta_{4}$ Management experience

$+\delta_{5}$ Planning

$+\delta_{6}$ Professional advice

$+\delta_{7}$ Educational level

$+\delta_{8}$ Staffing

$+\delta_{9}$ Product/service timing

$+\delta_{10}$ Economic timing

$+\delta_{11}$ Age of owner

$+\delta_{12}$ Partners

$+\delta_{13}$ Parents

$+\delta_{14}$ Minority

$+\delta_{15}$ Marketing skills $+\dot{\varepsilon}$

\section{Variable Measures}

The dependent variable is dichotomous (success or failure). Following prior studies methodology (see Hyder \& Lussier, 2016; Lussier et al., 2016; Lussier \& Halabi, 2010; Teng et al., 2011), this study uses profitability levels to categorize businesses into success or failure categories. Therefore, rural business is considered as a success if the profit level is average or above the industry average profit; and rural business is considered as a failure if the profit level is less than average profits. Using a 4-point scale, business owners are asked to select the appropriate level of profits, 4 - profit is above the industry profit, 3 - industry average profit, 2 - profit is below the industry average, and 1 - currently not making a profit. Entrepreneurs or business owners that selected 1 and 2 are coded as 0 to represent failure, and those that chose 3 and 4 are coded as 1 to represent success.

For the independent variables, the ratio number of years is used to measure three of the variables; industrial experience, managerial experience, and owner's age. A 7-point scale is used to measure nine variables: capital (1 adequate - 7 inadequate), record keeping and financial records (1 poor - 7 good), planning (1 specific -7 no plan), professional advice (1 used -7 not used), educational level (1 none -7 doctorate), staffing (1 difficult to recruit - 7 easy), product/service timing ( 1 introduction -7 decline), economic timing (1 expansion -7 recession) and marketing skills ( 1 unskilled - 7 skilled). Nominal measures coded as 1 or 2 are used for partners ( 1 owner -2 partner), parents ( 1 yes parent-owned business -2 no), and minority ( 1 foreigner/minority -2 Ghanaian).

\section{Results \\ Descriptive Statistics and Test of Differences of Lussier Model Variables}

Table 3 provides the descriptive and inferential statistics of the 15 model variables for 230 entrepreneurs in the rural communities in Ghana. T-test and chi-square are used to determine whether there are any significant $(p<0.05)$ differences between failed and successful businesses.

Of the 15 tests of differences, the successful rural businesses have 13 greater levels of resources than failed rural businesses. Though the failed rural businesses have 2 greater levels of resources (age and educational level of owners) than the successful rural businesses; the differences are not significant ( $p>0.05$ ). Thus, the small insignificant differences do not suggest waiting to start a business or that less education improves chances of success.

Five variables are significantly different at the .05 level, as discussed here. (1) Capital. Successful businesses in rural communities started with significantly greater capital $(\mathrm{m}=$ 4.39) than failed businesses $(m=5.85)$. As stated as a note under Table 3, capital is a reverse scale, and a lower value is preferable. (3) Years of industrial experience. The successful rural business owners have a significantly greater number of years of industrial experience $(m=10.92)$ before commencing their business than that of failed rural business owners (8.11). (4) Management experience. Successful rural business owners have a significantly greater number of years of managerial experience $(m=9.69)$ before starting their business than failed rural business owners $(m=7.69)$. (8) Staffing. The result shows that successful rural businesses have significantly fewer difficulties recruiting and retaining quality staff $(\mathrm{m}=6.59)$ than that of failed rural businesses $(\mathrm{m}=5.51)$. (15) Marketing skills. Successful rural business owners have significantly higher levels of marketing skills $(\mathrm{m}=4.91)$ before commencing their business than failed business owners $(m=4.25)$.

\section{Correlations}

The Pearson correlation matrix in Table 4 reports 105 correlations, and 33 (31\%) are significant correlations (p. $<.05)$ between variables. Part of the reason for the high rate of correlations is the sample size. The great the sample size, the more likely the r-value is to be significant, and vice versa (Lussier, 2005). The reason why some of the variables are significant is because of faced, near, or just multicollinearity. This happens when an independent variable depends linearly on one or more other independent variables, and without them, the estimate would not occur. For instance, industry experience, management experience, and age of owner are likely to be highly positively correlated. 
Table 3. Descriptive statistics and test of difference $(\mathrm{N}=230)$

\begin{tabular}{|c|c|c|c|c|}
\hline Model Variables & $\begin{array}{l}\text { Failed Mean }(n= \\
110)\end{array}$ & $\begin{array}{l}\text { Failed } \\
\text { S.D. }\end{array}$ & $\begin{array}{l}\text { Success Mean }(n= \\
120)\end{array}$ & $\begin{array}{l}\text { Success } \\
\text { S.D. }\end{array}$ \\
\hline $\begin{array}{l}\text { 1. Capital } \\
(1 \text { adequate }-7 \text { inadequate })^{a}\end{array}$ & 5.85 & 2.66 & 4.39 & $1.71^{* *}$ \\
\hline $\begin{array}{l}\text { 2. Record Keeping and Financial } \\
\text { (1 poor - } 7 \text { good) }\end{array}$ & 4.33 & 1.49 & 4.56 & 1.59 \\
\hline $\begin{array}{l}\text { 3. Industry Experience } \\
\text { (number of years) }\end{array}$ & 8.11 & 5.60 & 10.92 & $6.79^{* *}$ \\
\hline $\begin{array}{l}\text { 4. Management Experience } \\
\text { (number of years) }\end{array}$ & 7.69 & 5.39 & 9.69 & $6.43^{* *}$ \\
\hline $\begin{array}{l}\text { 5. Planning } \\
(1 \text { specific }-7 \text { no plan })^{a}\end{array}$ & 3.75 & 1.91 & 3.76 & 1.30 \\
\hline $\begin{array}{l}\text { 6. Professional Advice } \\
(1 \text { used }-7 \text { not used })^{a}\end{array}$ & 4.29 & 1.86 & 4.01 & 1.69 \\
\hline $\begin{array}{l}\text { 7. Education } \\
\text { (1 none }-7 \text { doctorate) }\end{array}$ & 1.86 & 0.86 & 1.75 & 0.73 \\
\hline $\begin{array}{l}\text { 8. Staffing } \\
\text { (1 difficult - } 7 \text { easy) }\end{array}$ & 5.51 & 1.22 & 6.59 & $3.78^{* *}$ \\
\hline $\begin{array}{l}\text { 9. Product/service Timing } \\
(1 \text { introduction }-7 \text { decline })^{a}\end{array}$ & 3.18 & 1.35 & 3.04 & 1.14 \\
\hline $\begin{array}{l}\text { 10. Economic Timing } \\
(1 \text { expansion }-7 \text { recession })^{a}\end{array}$ & 3.39 & 1.21 & 3.33 & 0.91 \\
\hline $\begin{array}{l}\text { 11. Age of Owner } \\
\text { (number of years) }\end{array}$ & 33.51 & 9.35 & 31.91 & 10.51 \\
\hline $\begin{array}{l}\text { 12. Partners } \\
\text { (1 owner } 49.5 \% \text { - } 2 \text { Partners } 50.5 \%)\end{array}$ & 1.20 & 0.41 & 1.23 & 0.41 \\
\hline $\begin{array}{l}\text { 13. Parents } \\
\text { ( } 1 \text { yes parent owned business } 45.7 \% 2 \text { no } \\
54.3 \%)\end{array}$ & 1.80 & 0.39 & 1.86 & 0.35 \\
\hline $\begin{array}{l}\text { 14. Minorities (Foreigners) } \\
\text { (1 yes } 15.9 \%-2 \text { no } 84.1 \% \text { ) }\end{array}$ & 1.96 & 0.18 & 1.99 & 0.09 \\
\hline $\begin{array}{l}\text { 15. Marketing } \\
\text { ( } 1 \text { unskilled }-7 \text { skilled) }\end{array}$ & 4.25 & 1.60 & 4.91 & $4.43^{*}$ \\
\hline
\end{tabular}

a Note that these are reverse scale items. Therefore, a lower number is preferred/expected.

Significance level $*$ p $<0.01 * \mathrm{p}<0.05$

Another important consideration is the issue of collinearity. R-values greater than $70 \%(r=0.700)$ indicate high collinearity that is problematic in regression models (Lussier, 2005). For the Pearson correlation matrix (Table 4 ), we found only one correlation greater than 0.700 ; (3) industry experience and (4) management experience $(\mathrm{r}=$ 0.95). As expected, industry and management experience are collinear since it is not likely to have several years of managerial experience without several years of industry experience (Lussier \& Pfeifer, 2000, 2001). Therefore, multicollinearity and collinearity issues should not be a problem for the study, and unlike a simple test of differences, running logistic regression addresses these issues. 
Table 4. Correlation matrix

\begin{tabular}{|c|c|c|c|c|c|c|c|c|c|c|c|c|c|c|}
\hline & 1 & 2 & 3 & 4 & 5 & 6 & 7 & 8 & 9 & 10 & 11 & 12 & 13 & 14 \\
\hline 1. Capital & 1.00 & & & & & & & & & & & & & \\
\hline $\begin{array}{l}\text { 2. Record keeping and } \\
\text { financial control }\end{array}$ & $-0.13^{* *}$ & 1.00 & & & & & & & & & & & & \\
\hline 3. Industry experience & 0.03 & $-0.29^{* *}$ & 1.00 & & & & & & & & & & & \\
\hline $\begin{array}{l}\text { 4. Management } \\
\text { experience }\end{array}$ & 0.03 & $-0.27^{* *}$ & $0.95^{* *}$ & 1.00 & & & & & & & & & & \\
\hline 5. Planning & 0.09 & -0.07 & 0.03 & 0.03 & 1.00 & & & & & & & & & \\
\hline 6. Professional advice & 0.08 & $-0.21^{* *}$ & $0.19^{* *}$ & $0.18^{* *}$ & $0.26^{* *}$ & 1.00 & & & & & & & & \\
\hline 7. Education & -0.05 & $0.18^{* *}$ & $-0.12^{*}$ & $-0.12^{*}$ & -0.08 & $-0.28^{* *}$ & 1.00 & & & & & & & \\
\hline 8. Staffing & -0.06 & 0.05 & 0.07 & 0.07 & 0.04 & 0.10 & $-0.14^{* *}$ & 1.00 & & & & & & \\
\hline $\begin{array}{l}\text { 9. Product/Service } \\
\text { timing }\end{array}$ & $-0.19^{* *}$ & 0.01 & -0.04 & -0.03 & -0.08 & $-0.11^{*}$ & -0.05 & -0.02 & 1.00 & & & & & \\
\hline 10. Economic timing & $0.18^{* *}$ & $-0.16^{* *}$ & $0.11^{*}$ & 0.07 & 0.08 & $0.18^{* *}$ & $-0.17^{* *}$ & -0.01 & 0.00 & 1.00 & & & & \\
\hline 11. Age of owner & 0.02 & -0.01 & $-0.32^{* *}$ & $-0.30^{* *}$ & 0.01 & -0.03 & $0.12^{*}$ & -0.02 & $0.14^{* *}$ & 0.01 & 1.00 & & & \\
\hline 12. Partners & 0.03 & $-0.14^{* *}$ & $0.14^{* *}$ & 0.10 & 0.07 & 0.02 & -0.06 & -0.04 & 0.11 & -0.06 & -0.07 & 1.00 & & \\
\hline 13. Parents & 0.02 & 0.07 & -0.10 & $-0.14^{* *}$ & -0.01 & -0.05 & 0.01 & 0.01 & -0.06 & 0.01 & $-0.13^{*}$ & $-0.13^{* *}$ & 1.00 & \\
\hline 14. Minority & 0.03 & -0.09 & 0.08 & 0.03 & 0.02 & 0.08 & 0.00 & 0.02 & 0.06 & $0.14^{* *}$ & $0.21^{* *}$ & 0.01 & 0.01 & 1.00 \\
\hline 15. Marketing skills & -0.11 & $0.12^{* *}$ & -0.01 & -0.04 & -0.06 & 0.04 & 0.04 & -0.01 & 0.01 & -0.07 & -0.03 & $0.18^{* *}$ & 0.03 & 0.03 \\
\hline
\end{tabular}

Significance level ${ }^{* * *} \mathrm{p}<0.01$ * $\mathrm{p}<0.05$ 
Table 5. Logistic regression model test results $(\mathrm{N}=230)$

\begin{tabular}{|c|c|c|c|}
\hline $\begin{array}{l}\text { Model Parameter Estimates } \\
\text { Variables Name }\end{array}$ & & $\begin{array}{c}\text { Model } \\
\boldsymbol{\beta}\end{array}$ & $\begin{array}{l}\text { Model } \\
\text { t-Sig. }\end{array}$ \\
\hline 1. Capital & & -0.205 & 0.034 \\
\hline 2. Record Keeping and Financial Control & & -0.138 & 0.229 \\
\hline 3. Industry Experience & & 0.197 & 0.041 \\
\hline 4. Management Experience & & -0.152 & 0.121 \\
\hline 5. Planning & & 0.067 & 0.547 \\
\hline 6. Professional Advice & & -0.032 & 0.733 \\
\hline 7. Education & & 0.825 & 0.693 \\
\hline 8. Staffing & & 0.934 & 0.000 \\
\hline 9. Product/Service Timing & & -0.127 & 0.293 \\
\hline 10. Economic Timing & & -0.004 & 0.405 \\
\hline 11. Age of Owner & & -0.073 & 0.812 \\
\hline 12. Partners & & 0.516 & 0.856 \\
\hline 13. Parents owned a business & & -0.567 & 0.234 \\
\hline 14. Minority & & 1.015 & 0.427 \\
\hline 15. Marketing & & 0.239 & 0.041 \\
\hline Constant & & -7.775 & 0.016 \\
\hline \multicolumn{4}{|l|}{ Model Test Results } \\
\hline -2 Log Likelihood & 257.424 & & \\
\hline Model Chi-square & 59.68 & & \\
\hline Model Significance & 0.000 & & \\
\hline Pseudo R Square & 0.1882 & & \\
\hline \multicolumn{4}{|l|}{ Classification Results } \\
\hline $\begin{array}{l}\text { Correctly Classified Cases } \\
\text { Success } \\
\text { Failed } \\
\text { Overall }\end{array}$ & $\begin{array}{l}71.20 \% \\
71.15 \% \\
71.18 \%\end{array}$ & & \\
\hline
\end{tabular}

\section{Logit Results of the Lussier Model}

Table 5 reports the logistic regression of the Lussier Model with a sample of 230 businesses from rural communities in an emerging market, Ghana. The appropriate test for the overall significance of the model is measured by a large -2 log-likelihood (LL) statistic, chi-square, and predictive power. The $-2 \log$ LL measures the goodness of fit of the model. Chi-square tests whether the combined effects of all the 15 variables in the model are different from zero. Thus, if the significant level is less than 0.05 (95\% confident interval), with a high accuracy rate, then the model's explanatory power is robust.

The results in Table 5 report the logistic regression results of the model -2 LL statistics 257.424 ( $\mathrm{p}=.000$ ), chisquared $59.68(\mathrm{p}=0.000)$, and overall accuracy rate of $71.8 \%$. This supports that the Lussier (1995) model is a valid predictor of the success or failure of rural businesses in emerging markets. The model is significant as it will predict a group of businesses as successful or failed $99 \%$ of the time, and the model will accurately classify a specific business as successful or failed greater than $70 \%$ of the time. Therefore, entrepreneurs in emerging markets that start with adequate capital, maintain good record keeping and financial control, have industrial and managerial experiences, have specific plans, seek professional advice, educated, have little difficulties recruiting and retaining quality workers, have better product or service and economic timing, have partners, have parents who owned businesses and have marketing skills before starting their businesses can increase their chances of success.

\section{Significant Variables}

There are four significant $t$-value variables $(p .<.05)$ that distinguish successful from failed businesses in the rural economy. The variables are: $(1)$ capital $(p=0.034)$, (4) industry experience $(\mathrm{p}=0.041)$, (8) staffing $(\mathrm{p}=0.000)$ and (15) marketing ( $p=0.041)$. The reduced model function for rural businesses in an emerging markets is:

$S=f$ (capital, industry experience,

staffing, and marketing skills).

The implication here is that these four variables are the most critical success factors for rural businesses in emerging markets. Thus, it is recommended that entrepreneurs should start with adequate capital, have industrial experi- 
ence, with the ability to attract and maintain quality staff, and should have marketing skills before starting their business.

\section{Discussion}

The findings of the study support the model validity of prior studies on businesses by Lussier (1995), Lussier \& Halabi (2010), Marom \& Lussier (2014). Guzman \& Lussier (2015), Hyder \& Lussier (2016), Lussier et al. (2016), and Baidoun et al. (2019). One of the most significant variables is capital that contributes to the success of rural businesses in emerging markets. Capital is also a significant contributing variable for the success of small businesses in Nigeria (Adeola et al., 2021; Gyimah \& Adeola, 2021), and other current studies by Baidoun et al. (2019) and Hyder \& Lussier (2016); as well as earlier studies by P. D. Reynolds (1987), P. Reynolds \& Miller (1989), Cooper et al. (1990), and Cooper et al. (1991).

The study also finds that the industry experience of entrepreneurs can increase the chances of success of rural businesses in emerging markets. Earlier studies by Barsley \& Kleiner (1990), Copper et al. (1991), G. L. Crawford (1974), Dun \& Bradstreet (1995), Flahvin (1985), Gaskill et al. (1993), Hoad \& Rosko (1964), Lussier \& Corman (1996), McQueen (1989), Rauch et al. (2005), Vesper (1990), and Wood (1989) also supported that entrepreneurs that have more years of experience in the industry increase their chances of success.

In terms of staffing, studies by Lussier (1996), Lussier \& Corman (1996), Lussier \& Pfeifer (2001), Hyder \& Lussier (2016), and Lussier et al. (2016); as well as earlier studies including Bruno et al. (1987), Flahvin (1985), Lauzen (1985), Sommers \& Koc (1987) and Thompson (1988) also found that quality staffing increases the success of businesses. Marketing skills, which is also is a predictor of the success of rural businesses in emerging markets, was also significant in earlier studies by Bruno et al. (1987), Gaskill et al. (1993), and McQueen (1989); as well as studies conducted by Lussier a (1996 a), Lussier et al. (2016) and Teng et al. (2011) that found that business owners with marketing skills before starting their business contributes significantly to the success of the business. Our result confirms prior studies outcomes but also highlights substantive gaps in capacity and resources for rural entrepreneurs, especially those in developing countries.

\section{Practical Implications}

The study examines the factors that contribute to the success or failure of small businesses in rural communities in an emerging market. Capital, industry experience, staffing, and marketing skills are the most significant (tvalues $<.05$ ) factors that distinguish successful from failed rural businesses in an emerging market. The study concludes that future and nascent rural entrepreneurs should use the model and start businesses with sufficient capital, have industrial experiences with adequate marketing skills, and have qualified staff to increase the chance of being successful. This can help future, and nascent rural entrepreneurs avoid failure and successfully contribute to economic development.
Moreover, public advocacy organizations in Ghana, such as National Board for Small Scale Industries (NBSSI) and Microfinance and Small Loans Centre (MASLOC), and other NGOs working directly in the rural communities should provide capital support by giving low-interest credits or loans to rural entrepreneurs. The ongoing public-private partnership policy on "The one district one factory" initiative in Ghana should focus on rural enterprises since they contribute massively to the UN Sustainable Development Goals of poverty reduction (SDG 1) and good jobs and economic growth (SDG 8). Again, government agencies in developing economies can use the model to assist them in providing training and resources to successful rural businesses and for new ventures that have the potential to be successful so that they can contribute to economic development.

Government should also provide industrial mentors with vast industrial experiences to rural entrepreneurs to help grow, expand, multiply, and replicate their businesses. Likewise, Business Advisory Centres (BACs) of NBSSI in the local communities in Ghana should organize seminars on how to support entrepreneurs in marketing their products or services that contribute to economic development.

Furthermore, educational and professional institutions should teach the model and key success factors behind rural entrepreneurship in emerging markets. For instance, technical and vocational education and training (TVET) institutions, senior high schools, training colleges, and universities in developing countries should use the validated Lussier model to teach apprentices or learners about the drivers of rural entrepreneurship. Lussier's success vs failure prediction model should be included in entrepreneurship programs and seminars of professional and educational or training institutions.

Additionally, potential investors or future rural entrepreneurs can use the model to evaluate investment decisions. Through the entrepreneurial use of the model, society can benefit directly through job creation and indirectly through local economic growth and development. Tax revenues from new ventures can be used by the government to invest in infrastructure to future develop the needed infrastructure to stimulate continuing economic growth. Additionally, public regulatory bodies and policymakers should keep stimulating entrepreneurship in times of economic downturn.

Current and prior bankruptcy and default prediction models are usually based on historical financial records or performance. Nascent and future ventures have no prior history, and this strengthens the value of the Lussier (1995) model that can be used to evaluate the success or failure of rural businesses in other emerging and developed markets.

\section{Limitations and Suggestions for Further Research}

As with all research, there are limitations to this study, so we present limitations here with suggestions on how to improve on these limitations in further research. First, the study only uses a Ghanaian sample to represent rural businesses in emerging markets, excluding other emerging or developing countries. Future studies can duplicate this study using datasets from other emerging countries to im- 
prove the generalizability of the findings in predicting the success or failure of rural businesses in emerging markets.

Another limitation is the measure of the dichotomous dependent variable (success or failure), where the profitability level is used to categorized failed and successful rural businesses. Entrepreneurs must make a subjective judgement on their level of profitability. A similar approach by prior studies by Adeola et al. (2021), Hyder \& Lussier (2016), Lussier \& Halabi (2010), Lussier et al. (2016), and Teng et al. (2011) used the profitability levels due to lack of records of failed businesses. Although using profitability is an acceptable methodology when there is a lack of sample information, future studies are encouraged to use the more robust methodology in the original Lussier (1995) study. Lussier used a two-step sampling process. First, government public record information on Chapter 11 and bankruptcy was obtained to identify failed businesses. Step one of data collection surveyed failed business owners. The second sampling of successful firms used a matched pairs sampling by locating a successful business in the same industry and area matching it with a previous respondent failed business that completed the survey. Thus, the total sample had the same number of successful and failed businesses from the same industry and local area of the country.

The subjective measure of the independent variables is another limitation. A 7-point scale is used to measure nine of Lussier's model variables. So judgment is needed, such as rating each variable as high, medium, or low to determine its effect on success or failure. Also, when using the model, entrepreneurs are subject to self-perception bias responses that can lead to over-rating the chances of success that can fail. Thus, further research should consider developing more objective indicators for measuring the model independent variables, and additional variables can be added to make the model more robust.

Stakeholders such as government agencies, public regulatory bodies, financial institutions, investors, suppliers, educators, and professional institutions, and entrepreneurs can use the model to evaluate the failure or success of new and nascent businesses. However, based on its subjectivity in the judgement of variable measurement, it is better to use other prediction methods in addition to the Lussier model when making decisions to improve the accuracy of the prediction.

Additionally, the study excludes cultural, regulatory requirements, and economic factors that can affect the success or failure of rural businesses in various countries. Further research can include those indicator factors as moderating or mediating or control variables to assess their effect on the probability of success or failure of businesses.

\section{Conclusion}

This study statistically tested the Lussier success vs failure prediction model in a rural developing country. The logistic regression results are significant $(p=.000)$, and the model will predict a group of businesses as successful or failed $99 \%$ of the time, and the model will accurately classify a specific business as successful or failed greater than $70 \%$ of the time. This study contributes to the international validity of the Lussier model that can be used in both advanced and developing economies, and it contributes to the development of theory. 


\section{REFERENCES}

Abebe, G. K., \& Gebremariam, T. A. (2021). Challenges for entrepreneurship development in rural economies: The case of micro and small-scale enterprises in Ethiopia. Small Enterprise Research, 28(1), 36-56. https://doi.org/10.1080/13215906.202 $\underline{1.1878385}$

Achua, C. F., \& Lussier, R. N. (2014). Entrepreneurial drive and the informal economy in Cameroon. Journal of Developmental Entrepreneurship, 19(4), 1-12. http s://doi.org/10.1142/s1084946714500241

Adeola, O., Gyimah, P., Appiah, K. O., \& Lussier, R. N. (2021). Can critical success factors of small businesses in emerging markets advance UN Sustainable Development Goals? World Journal of Entrepreneurship, Management and Sustainable Development, 17(1), 85-105. https://doi.org/10.1108/ wjemsd-09-2019-0072

Appiah, K. O. (2011). Corporate failure prediction: Some empirical evidence from listed firms in Ghana. ChinaUSA Business Review, 10(1), 32-41.

Appiah, K. O., Chizema, A., \& Arthur, J. (2015). Predicting corporate failure: A systematic literature review of methodological issues. International Journal of Law and Management, 57(5), 461-485. https://doi.o rg/10.1108/ijlma-04-2014-0032

Baidoun, S. D., Lussier, R. N., Burbar, M., \& Awashra, S. (2019). Prediction model of business success or failure for Palestinian small enterprises in the West Bank. Journal of Entrepreneurship in Emerging Economies, 10(1), 60-80. https://doi.org/10.1108/jee e-02-2017-0013

Barber, D., III, Saadatmand, Y., \& Pierce, J. (2019). Does gender inequality matter for female entrepreneurial activities? An investigation of selected OECD countries. Small Business Institute Journal, 15(1), 103-124.

Barkham, R., Gudgin, G., Hart, M., \& Hanvey, E. (1996). The Determinants of Small Firm Growth: An Interregional Study in the United Kingdom 1986-1990. Jessica Kingsley.

Barsley, G., \& Kleiner, B. H. (1990). Small business management: Ensuring your clients success. National Public Accountant, 35(2), 30-33.

Benzing, C., Chu, H. M., \& Callanan, G. (2005). A regional comparison of the motivation and problems of Vietnamese entrepreneurs. Journal of Developmental Entrepreneurship, 10(1), 3-27. https://d oi.org/10.1142/s1084946705000033

Blackwood, T., \& Mowl, G. (2000). Expatriate-owned small businesses: Measuring and accounting for success. International Small Business Journal, 18(3), 60-73. https://doi.org/10.1177/0266242600183004

Blankson, C., Cowan, K., \& Darley, W. K. (2018). Marketing practices of rural micro and small businesses in Ghana: The role of public policy. Journal of Macromarketing, 38(1), 29-56. https://doi.org/10.11 $\underline{77 / 0276146717741067}$
Boohene, R., \& Agyapong, D. (2017). Rural entrepreneurship in African countries: A synthesis of related literature. Journal of Small Business and Entrepreneurship Development, 5(1), 43-54. https://do i.org/10.15640/jsbed.v5n1a5

Borchardt, M., Pereira, G., Viegas, C., Reolon, D., Xavier, Y., \& Battaglia, D. (2018). Providing goods to the base of the pyramid: Opportunities for micro, small and medium-sized local producers. Journal of Small Business Strategy, 28(2), 80-89.

Bosma, N., Van Praag, M., \& De Wit, G. (2000). Determinants of successful entrepreneurship. Research Report, 0002, 1-42.

Bruins, A., op de Coul, J., Stigter, H. W., \& van Uxem, F. W. (2000). What determines the success of a starter? Analysis of Success and Failure Factors of Entrepreneurs. Research Report, Research Institute for Small and Medium-sized Business in the Netherlands, Zoetermeer.

Bruno, A. V., Leidecker, J. K., \& Harder, J. W. (1987). Why Firms Fail. Business Horizons, 36(2), 50-58. http s://doi.org/10.1016/0007-6813(87)90009-7

Chu, H. M., Benzing, C., \& McGee, C. (2007). Ghanaian and Kenyan entrepreneurs: A comparative analysis of their motivations, success characteristics and problems. Journal of Developmental Entrepreneurship, 12(3), 295-322. https://doi.org/10.1142/s1084946707 000691

Ciampi, F., Giannozzi, A., Marzi, G., \& Altman, E. I. (2021). Rethinking SME default prediction: A systematic literature review and future perspectives. Scientometrics, 126(3), 2141-2188. https://doi.org/1 0.1007/s11192-020-03856-0

Cooper, A. C., Dunkelberg, W., Woo, C. Y., \& Dennis, W. (1990). New Business in America: The Firms \& Their Owners. Washington, DC, the NFIB Foundation.

Cooper, A. C., Gimeno-Gascon, F. J., \& Woo, C. Y. (1991). A resource-based prediction of new venture survival and growth. Academy of Management Proceedings, 1991(1), 68-72. https://doi.org/10.5465/a mbpp.1991.4976561

Covin, J. G., \& Covin, T. J. (1990). Competitive aggressiveness, environmental context, and small firm performance. Entrepreneurship Theory and Practice, 14(4), 35-50. https://doi.org/10.1177/104225 $\underline{879001400406}$

Crawford, A., \& Barber, D., III. (2020). The gap in transition planning education opportunities for rural entrepreneurs. American Journal of Entrepreneurship, 13(1), 92-125.

Crawford, G. L. (1974). An Analysis of Management Factors That May Contribute to the Success or Failure of Selected Small Retailers. University of Arkansas.

Deller, S. C., \& Conroy, T. (2017). Business survival rates across the urban-rural divide. Community Development, 48(1), 67-85. https://doi.org/10.1080/15 $\underline{575330.2016 .1246459}$ 
Deller, S. C., Kures, M., \& Conroy, T. (2019). Rural entrepreneurship and migration. Journal of Rural Studies, 66, 30-42. https://doi.org/10.1016/j.jrurstu d.2019.01.026

Dennis, W. J., Jr., \& Fernald, L. W., Jr. (2001). The chances of financial success (and Loss) from small business ownership. Entrepreneurship: Theory and Practice, 26(1), 75-83.

Dess, G. G., Lumpkin, G. T., \& Covin, J. G. (1997). Entrepreneurial strategy making and firm performance: Tests of contingency and configurational models. Strategic Management Journal, 18(9), 677-695. https://doi.org/10.1002/(sic i)1097-0266(199710)18:9

Duchesneau, D. A., \& Gartner, W. B. (1990). A profile of new venture success and failure in an emerging industry. Journal of Business Venturing, 5(5), 297-312. https://doi.org/10.1016/0883-9026(90)90007-g

Dun \& Bradstreet. (1995). Business Failure Record. Dun \& Bradstreet.

Dunne, T. C., Aaron, J. R., McDowell, W. C., Urban, D. J., \& Geho, P. R. (2016). The impact of leadership on small business innovativeness. Journal of Business Research, 69(11), 4876-4881. https://doi.org/10.1016/ j.jbusres.2016.04.046

Flahvin, A. (1985). Why small businesses fail. Australian Accountant, 55(9), 56-72.

Fortunato, M. W.-P. (2014). Supporting rural entrepreneurship: A review of conceptual developments from research to practice. Community Development, 45(4), 387-408. https://doi.org/10.1080/ 15575330.2014 .935795

Galli-Debicella, A. (2020). The efficacy of SBA loans on small firm survival rates. Journal of Small Business Strategy, 30(2), 26-34.

Gaskill, L. R., Van Auken, H. E., \& Manning, R. A. (1993). A factor analytic study of the perceived causes of small business failure. Journal of Small Business Management, 31(4), 18.

Ghosh, B. C., Kim, T. S., \& Meng, L. A. (1993). Factors contributing to the success of local SMEs: An insight from Singapore. Journal of Small Business \& Entrepreneurship, 10(3), 33-46. https://doi.org/10.108 $\underline{0 / 08276331.1993 .10600426}$

Guzman, J. B., \& Lussier, R. N. (2015). Success factors for small businesses in Guanajuato, Mexico. International Journal of Business and Social Science, 6(11), 1-7.

Gyimah, P., \& Adeola, O. (2021). MSMEs sustainable prediction model: A three-sector comparative study. Journal of the International Council for Small Business, 2(2), 90-100. https://doi.org/10.1080/26437015.202 $\underline{1.1881933}$

Gyimah, P., Appiah, K. O., \& Lussier, R. N. (2020). Success versus failure prediction model for small businesses in Ghana. Journal of African Business, 21(2), 215-234. https://doi.org/10.1080/15228916.201 $\underline{9.1625017}$
Gyimah, P., \& Boachie, W. K. (2018). Portability of multiple discriminant analysis prediction model of listed firms: An emerging market perspective. Research Journal of Accounting and Finance, 6(6), 94-99.

Gyimah, P., Marom, S., \& Lussier, R. N. (2019). Small business success or failure prediction: A comparative study in Ghana and Israel. Journal of Applied Business and Economics, 21(3), 37-52.

Habersetzer, A., Rataj, M., Eriksson, R. H., \& Mayer, H. (2021). Entrepreneurship in rural regions: The role of industry experience and home advantage for newly founded firms. Regional Studies, 55(5), 936-950. http s://doi.org/10.1080/00343404.2020.1826038

Halabí, C. E., \& Lussier, R. N. (2014). A model for predicting small firm performance: Increasing the probability of entrepreneurial success in Chile. Journal of Small Business and Enterprise Development, 21(1), 4-25. https://doi.org/10.1108/isbed-10-2013-0 $\underline{141}$

Hoad, W. M., \& Rosko, P. (1964). Management Factors Contributing to the Success or Failure of New Small Manufacturers (Vol. 41). Bureau of Business Research, Graduate School of Business Administration, University of Michigan.

Huck, J. F., \& McEwen, T. (1991). Competencies needed for small business success: Perceptions. Journal of Small Business Management, 29(4), 90.

Hyder, S., \& Lussier, R. N. (2016). Why businesses succeed or fail: A study on small businesses in Pakistan. Journal of Entrepreneurship in Emerging Economies, 8(1), 82-100. https://doi.org/10.1108/jee e-03-2015-0020

Kangasharju, A. (2000). Growth of the smallest: Determinants of small firm growth during strong macroeconomic fluctuations. International Small Business Journal, 19(1), 28-43. https://doi.org/10.117 7/0266242600191002

Korsgaard, S., Müller, S., \& Tanvig, H. W. (2015). Rural entrepreneurship or entrepreneurship in the rural between place and space. International Journal of Entrepreneurial Behavior \& Research, 21(1), 5-26. http s://doi.org/10.1108/ijebr-11-2013-0205

Koyana, S., \& Mason, R. B. (2017). Rural entrepreneurship and transformation: The role of learnerships. International Journal of Entrepreneurial Behavior \& Research, 23(5), 734-751. https://doi.org/1 0.1108/ijebr-07-2016-0207

Lauzen, L. (1985). Small business failures are controllable. Corporate Accounting, 3(3), 34-38.

Lussier, R. N. (1995). A non-financial business success versus failure prediction model. Journal of Small Business Management, 33(1), 8-20.

Lussier, R. N. (1996). A business success versus failure prediction model for service industries. Journal of Business and Entrepreneurship, 8(2), 23-37.

Lussier, R. N. (2005). A success versus failure prediction model for the real estate industry. American Journal of Business, 20(1), 47-53. https://doi.org/10.1108/19355 $\underline{181200500005}$ 
Lussier, R. N., Bandara, C., \& Marom, S. (2016). Entrepreneurship success factors: An empirical investigation in Sri Lanka. World Journal of Entrepreneurship, Management and Sustainable Development, 12(2), 102-112. https://doi.org/10.1108/ wjemsd-10-2015-0047

Lussier, R. N., \& Corman, J. (1995). There are few differences between successful and failed small businesses. Journal of Small Business Strategy, 6(1), 21-34.

Lussier, R. N., \& Corman, J. (1996). A business success versus failure prediction model for entrepreneurs with 0-10 employees. Journal of Small Business Strategy, 7(1), 21-36.

Lussier, R. N., \& Halabi, C. E. (2010). A Three-Country Comparison of the Business Success versus Failure Prediction Model. Journal of Small Business Management, 48(3), 360-377. https://doi.org/10.1111/ j.1540-627x.2010.00298.x

Lussier, R. N., \& Pfeifer, S. (2000). A comparison of business success versus failure variables between U.S. and Central Eastern Europe Croatian entrepreneurs. Entrepreneurship Theory and Practice, 24(4), 59-67.

Lussier, R. N., \& Pfeifer, S. (2001). A cross-national prediction model for business success. Journal of Small Business Management, 39(3), 228-239. https://d oi.org/10.1111/0447-2778.00021

Marom, S., \& Lussier, R. N. (2014). A business success versus failure prediction model for small businesses in Israel. Business and Economic Research, 4(2), 63-81. https://doi.org/10.5296/ber.v4i2.5997

McElwee, G., \& Smith, R. (2014). Researching rural enterprise. In A. Fayolle (Ed.), Handbook of Research on Entrepreneurship (pp. 307-334). Edward Elgar.

McQueen, J. (1989). The causes and lessons of business failure. Credit Management, 10, 24-25.

Miskin, V., \& Rose, J. (1990). New venture initiation: Factors influencing success. Journal of Small Business Strategy, 1(2), 1-9.

Müller, S., \& Korsgaard, S. (2018). Resources and bridging: The role of spatial context in rural entrepreneurship. Entrepreneurship \& Regional Development, 30(1-2), 224-255. https://doi.org/10.10 $\underline{80 / 08985626.2017 .1402092}$

Muñoz, P., \& Kimmitt, J. (2019). Rural entrepreneurship in place: An integrated framework. Entrepreneurship \& Regional Development, 31(9-10), 842-873. https://d oi.org/10.1080/08985626.2019.1609593

Neshamba, F. (2000). Growth and Transformation among Small Business in Kenya. The Nottingham Trent University.

Nikolić, N., Jovanović, I., Nikolić, Đ., Mihajlović, I., \& Schulte, P. (2019). Investigation of the factors influencing SME failure as a function of its prevention and fast recovery after failure. Entrepreneurship Research Journal, 9(3), 1-21. https://doi.org/10.1515/e ri-2017-0030

Oppong, M., Owiredu, A., \& Churchill, R. Q. (2014). Micro and small scale enterprises development in Ghana. European Journal of Accounting Auditing and Finance Research, 2(6), 84-97.
Pato, L., \& Teixeira, A. A. C. (2018). Rural entrepreneurship: The tale of a rare event. Journal of Place Management and Development, 11(1), 46-59. htt ps://doi.org/10.1108/jpmd-08-2017-0085

Paynter, S. R., Harris, M. L., \& Barber, D., III. (2021). Regional advisory councils to support nascent rural entrepreneurs. Journal of Extension, 58(3), 5.

Peake, W. O., Barber, D., III, McMilan, A., Bolton, D. L., \& Coder, L. (2019). Do management control systems stifle innovation in small firms? A mediation approach. Journal of Small Business Strategy, 29(2), $1-21$.

Postma, T. J., \& Zwart, P. S. (2001). Strategic research and performance of SMEs'. Journal of Small Business Strategy, 12(2), 52-64.

Rauch, A., Frese, M., \& Utsch, A. (2005). Effects of human capital and long - term human resources development and utilization on employment growth of small - scale businesses: A causal analysis. Entrepreneurship Theory and Practice, 29(6), 681-698.

Reynolds, P. D. (1987). New firms: Societal contribution versus survival potential. Journal of Business Venturing, 2(3), 231-246. https://doi.org/10.1016/088 3-9026(87)90011-5

Reynolds, P., \& Miller, B. (1989). New firm survival: Analysis of a panel's fourth year. Frontiers of Entrepreneurship Research, 159-172.

Rosner, R. L. (2003). Earnings manipulation in failing firms. Contemporary Accounting Research, 20(2), 361-408. https://doi.org/10.1506/8evn-9krb-3ae4-ee8 1

Saadatmand, Y., \& Barber, D., III. (2019). Globalization, gender and entrepreneurial activities in Latin America. Journal of Developmental Entrepreneurship, 24(04), 1950024. https://doi.org/10.1142/s1084946719 $\underline{500249}$

Salder, J., Gilman, M., Raby, S., \& Gkikas, A. (2020). Beyond linearity and resource-based perspectives of SME growth. Journal of Small Business Strategy, 30(1), $1-17$.

Skuras, D., Meccheri, N., Moreira, M. B., Rosell, J., \& Stathopoulou, S. (2005). Entrepreneurial human capital accumulation and the growth of rural businesses: A four-country survey in mountainous and lagging areas of the European union. Journal of Rural Studies, 21(1), 67-79. https://doi.org/10.1016/i.j rurstud.2004.05.001

Soleymani, A., Farani, A. Y., Karimi, S., Azadi, H., Nadiri, H., \& Scheffran, J. (2021). Identifying sustainable rural entrepreneurship indicators in the Iranian context. Journal of Cleaner Production, 290, 125186. https://doi.org/10.1016/i.jclepro.2020.125186

Sommers, W. P., \& Koc, A. (1987). Why most new ventures fail (and how others don't). Management Review, 76(9), 35.

Storey, D. J. (1994). New firm growth and bank financing. Small Business Economics, 6(2), 139-150. ht tps://doi.org/10.1007/bf01065186 
Teng, H. S. S., Bhatia, G. S., \& Anwar, S. (2011). A success versus failure prediction model for small businesses in Singapore. American Journal of Business, 26(1), 50-64. https://doi.org/10.1108/1935518111112 $\underline{4106}$

Thompson, R. (1988). Business plans: Myth and reality. Nation's Business, 76(8), 16-23.

Vesper, K. (1990). New Venture Strategies. Prentice Hall.

Wood, D. L. (1989). Why new businesses fail and how to avoid disaster. Corporate Cash Flow, 10(8), 26-27.

Wortman, M. S., Jr. (1990). Rural entrepreneurship research: An integration into the entrepreneurship field. Agribusiness, 6(4), 329-344. https://doi.org/10.1 002/1520-6297(199007)6:4
Yeboah-Asiamah, E., Musah-Surugu, I. J., Bawole, J. N., \& Nurudeen, S. (2015). The 'third sector' and poverty reduction in developing societies: The experience of hunger project in selected rural communities of Ghana. International Journal of Rural Management, 11(2), 85-110. https://doi.org/10.1177/097300521560 $\underline{1944}$

Yusuf, A. (1995). Critical success factors for small business: Perceptions of South Pacific entrepreneurs. Journal of Small Business Management, 33(2), 68. 